OPEN ACCESS

Edited by:

Quirino Lai,

Sapienza University of Rome, Italy

Reviewed by:

Alexander H. Petter-Puchner, Institute for Experimental and Clinical Traumatology (LBG), Austria

Claudio Cannistra

Hôpital

Bichat-Claude-Bernard, France

${ }^{*}$ Correspondence: Salvatore Sorrent salvatore.sorrenti@uniroma1.it

tThese authors have contributed equally to this work

Specialty section

This article was submitted to Surgical Oncology,

a section of the journal

Frontiers in Oncology

Received: 10 April 2020 Accepted: 28 May 2020 Published: 17 July 2020

Citation:

De Luca A, Tripodi D, Frusone $F$ Leonardi B, Cerbelli B, Botticelli $A$, Vergine $M, D$ 'Andrea V, Pironi $D$,

Sorrenti S and Amabile MI (2020)

Retrospective Evaluation of the Effectiveness of a Synthetic Glue and a Fibrin-Based Sealant for the Prevention of Seroma Following Axillary Dissection in Breast Cancer

Patients. Front. Oncol. 10:1061. doi: 10.3389/fonc.2020.01061

\section{Retrospective Evaluation of the Effectiveness of a Synthetic Glue and a Fibrin-Based Sealant for the Prevention of Seroma Following Axillary Dissection in Breast Cancer Patients}

\author{
Alessandro De Luca ${ }^{1 \dagger}$, Domenico Tripodi ${ }^{1+}$, Federico Frusone ${ }^{1}$, Beatrice Leonardi ${ }^{1}$, \\ Bruna Cerbelli ${ }^{2}$, Andrea Botticelli ${ }^{3}$, Massimo Vergine ${ }^{1}$, Vito D'Andrea ${ }^{1}$, Daniele Pironi ${ }^{1}$, \\ Salvatore Sorrenti ${ }^{1 *}$ and Maria Ida Amabile ${ }^{1}$ \\ ${ }^{1}$ Department of Surgical Sciences, Sapienza University of Rome, Rome, Italy, ${ }^{2}$ Department of Medico-Surgical Sciences and \\ Biotechnologies, Sapienza University of Rome, Rome, Italy, ${ }^{3}$ Department of Clinical and Molecular Medicine, Sapienza \\ University of Rome, Rome, Italy
}

Introduction: Seroma formation represents one of the most frequent postoperative complications of axillary dissection in breast cancer (BC) patients. We aimed to retrospectively explore the effectiveness of the intraoperative use of a synthetic cyanoacrylate glue (specifically Glubran®2) vs. the intraoperative use of a fibrin sealant (specifically Tisseel) in reducing seroma formation compared to the use of nonsealant in $\mathrm{BC}$ patients who underwent breast surgery and axillary dissection.

Materials and Methods: We conducted a retrospective, monocentric observational study on BC patients who underwent axillary dissection associated with breast surgery. The axillary dissection was completed with the application of a closed suction drain and was preceded by the application of either Glubran $® 2$ glue or Tisseel sealant or nonsealant. We analyzed the quantity of serum drained in the first 3 postoperative days, length of hospitalization, days of permanence of axillary drain, seroma development, and presence of postoperative infection signs.

Results: Forty-one BC patients were considered. Based on the device used during the surgical treatment, the patients were divided into three groups: group A (17 patients), to whom suction axillary drain was applied; group B (7 patients), to whom Tisseel and axillary suction drain were applied; and group C (17 patients), to whom Glubran ${ }^{2}$ and axillary suction drain were applied. Among the three groups, we did not find significant differences in terms of amount of serum drained in the first 3 postoperative days, length of hospitalization, and incidence of seroma. Group C maintained the axillary drain in a significantly lower number of days compared to the other two groups $(p=0.02)$; it also had a lower incidence of postoperative infections (6\%) compared to group A (23\%) and group B $(57 \%) \quad(p=0.02)$. 
Conclusions: We did not find any evidence that the use of surgical glues may reduce the formation of seroma following axillary dissection in BC patients. Nevertheless, the use of cyanoacrylate glue in association with closed suction axillary drain seems to contribute to the reduction in days of axillary drain permanence and of postoperative infections, which are known factors delaying the schedule of any adjuvant oncological therapies.

Keywords: cyanoacrylate glue, fibrin sealant, breast cancer, axillary dissection, seroma

\section{INTRODUCTION}

Breast cancer (BC) surgical treatment includes conservative or demolitive procedures associated to axillary staging surgery consisting of sentinel node biopsy and/or axillary dissection (13 ). It has been shown that a frequent complication of these types of treatments is represented by seroma development (4), with a percentage ranging from 15 to $85 \%$ (5), together with infection of the surgical site and bleeding. Both breast and axillary surgery, due to the dissection of small lymphatic and blood vessels, determine the formation of a highly suitable environment for the collection of fluids, although its pathophysiological process is not completely known (6).

The seroma may lead to dehiscence of the surgical wound and necrosis of the skin flaps, further increasing the risk of infection and reoperation, as well as interfering with the recovery of upper limb mobility and delaying the start of any adjuvant therapies $(4,7)$. Moreover, in the case of seroma, its evacuation through repeated transcutaneous aspirations may increase patient's discomfort and delay the return to common domestic and daily activities (7).

Several techniques have been tested over time to prevent the formation of seroma, i.e., use of continuous suction drainages (8), topical application of tetracyclines (9), external compression bandages (10), and use of dissectors with ultrasound technology (11). In addition, the use of fibrin sealant has presented interesting results because of its adhesive and hemostatic properties and in part also for its potential biostimulating ability for tissue regeneration $(12,13)$; in particular, its combination with "quilting" sutures has been documented to significantly reduce total drainage, hospital stay, and postoperative seroma incidence in patients who underwent postmastectomy breast reconstruction with dorsal muscle flap (14). Interesting results in reducing lymphatic production and seroma development after demolitive and/or conservative breast surgery and after pelvic lymphadenectomy were also obtained using a cyanoacrylatebased synthetic surgical glue $(7,15)$. In particular, recently, the use of a specific type of cyanoacrylate-based synthetic adhesive, Glubran ${ }^{\circledR} 2$, developed to minimize toxic effect of cyanoacrylate glues, has been described in surgery in substitution or in addition to sutures or staples for its adhesive properties and for the potential activity in decreasing activated partial thromboplastin time, exerting a hemostatic effect and therefore further contributing to tissue adhesion (16-18).

In this light, we aimed to retrospectively explore the effectiveness of the intraoperative use of a synthetic cyanoacrylate glue (specifically Glubran ${ }^{\circledR}$ ) vs. the intraoperative use of a fibrin sealant (specifically Tisseel) in reducing seroma formation compared to the use of nonsealant in $\mathrm{BC}$ patients who underwent breast surgery and axillary dissection.

\section{MATERIALS AND METHODS}

\section{Patients}

This is a retrospective, monocentric study conducted on patients diagnosed with BC and treated at the Department of Surgical Sciences, Sapienza University of Rome. The study was approved by the local Ethics Committee. All procedures were in accordance with the ethical standards of the Helsinki Declaration issued in 1975 and later amendments. We considered patients who underwent axillary dissection associated with mastectomy or breast conservative surgery. The surgical indication to axillary dissection was placed during the multidisciplinary meeting in patients with clinically positive axillary lymph nodes and cytological exam, following lymph node aspiration, positive for neoplastic cells, or in cases of sentinel node biopsy positive for the presence of macrometastasis.

The following clinical data were collected:

- age, weight, height, body mass index (BMI);

- immunohistochemical features of the tumor, radiological staging of axillary lymph nodes, eventual neoadjuvant chemotherapy performed, type of surgery performed, tumor-nodes-metastasis (TNM) staging, number of lymph nodes removed;

- length of hospital stay, daily volume of the drain content during the days of hospitalization, number of days of drain permanence at the surgical site, eventual seroma development and its aspiration, fever, infection, and/or dehiscence of the surgical wound.

\section{Surgical Procedure}

The patients underwent surgery under general anesthesia. During axillary lymph nodes dissection, the lymphatic vessels afferent to the removed lymph nodes were tied, and the long and thoracodorsal thoracic nerves were preserved. Clips and electrocautery were used to achieve hemostasis and lymphostasis. Once the axillary lymph nodes were removed and hemostasis performed, the surgical procedure was completed with the position of a suction drain preceded by the application of a fibrin sealant (Tisseel, $2 \mathrm{ml}$ ), nebulized on the surgical site, or by the application of a cyanoacrylate glue (Glubran $\AA 2,1 \mathrm{ml}$ ), nebulized with a dedicated system on the surgical site. There were cases conducted only with the suction drain position without the 
application of any sealant. The wound was then medicated and an elastocompression bandage performed.

\section{Fibrin Sealant and Synthetic Cyanoacrylate Glue}

Tisseel low-dose fibrin sealant, a human-derived local hemostatic drug consisting of a solution of two components, human fibrinogen, and human thrombin, was sprayed on all visible surgical surfaces in a thin layer. Contraidnications to the use of fibrin sealant were hypersensitivity to the components or to any of the excipients and intravascular use.

The Glubran $\AA 2$ glue, a surgical cyanoacrylate glue of synthetic origin (combination of n-butyl cyanoacrylate and methacryloxysulfolane monomers) for internal and endovascular use, was nebulized in a small amount on the surgical site with a dedicated pressure regulator system to allow its application on all visible surfaces in a thin layer. Contraindications to the use of cyanoacrylate adhesive were hypersensitivity of the subjects to the components or to any of the excipients, and pregnancy.

\section{Postoperative Period Care}

The compressive bandage was removed after $48 \mathrm{~h}$, and patients were discharged in the second to third postoperative day, except in cases of complications and/or comorbidities.

Suction drain was checked and emptied daily during hospitalization, and the amount of serum collected was recorded. Following discharge, the patients were reviewed in an outpatient setting and the quantity of drained serum measured.

The drain was removed when the quantity of serum collected was $<40 \mathrm{ml} /$ day. In case of axillary seroma development after removal of the drain, its aspiration was evaluated and recorded. The decision to aspirate a seroma was based on the assessment of its volume and the discomfort reported by the patient.

Local or general signs of infection was also assessed and antibiotic therapy prescribed as needed.

\section{Statistical Analysis}

Data regarding the quantity of serum drained in the first 3 postoperative days, duration of hospitalization, days of permanence of axillary drainage, development of seroma, and presence of signs of infection were collected. Patients' characteristics were described using mean \pm standard deviation (SD) for continuous normally distributed variables.

For the comparison between groups, one-way ANOVA test was used for continuous variables and the chi-square test for categorical variables.

A $p<0.05$ was considered statistically significant. The statistical analysis was performed with IBM SPSS Statistics 25.

\section{RESULTS}

\section{Patients' Characteristics}

Forty-one BC patients who underwent axillary dissection associated with mastectomy or breast conservative surgery were considered.

All patients received diagnosis of invasive ductal or lobular BC obtained by radiological guided breast needle biopsy.
Based on the device used during the surgical treatment, the patients were divided into three groups:

- group A, consisting of 17 patients, to whom axillary closed suction drain had been placed in the axillary fossa;

- group B, consisting of 7 patients, to whom Tisseel sealant and axillary closed suction drain had been placed in the axillary fossa;

- group C, consisting of 17 patients, to whom Glubran $\AA 2$ glue and axillary closed suction drain had been placed in the axillary fossa.

The mean age of the patients was $58 \pm 14.2$ years, and the mean BMI was $25 \pm 4.86$. Patients' characteristics of each group are shown in the Table 1. Ten patients had diabetes mellitus, and 16 patients reported dyslipidemia in past medical history.

The BC definitive histological diagnosis was laid for ductal invasive carcinoma in $83 \%$ of the patients, lobular invasive carcinoma in $14.6 \%$, and metaplastic carcinoma in $2.4 \%$ (one case) (Table 1).

The average size of the breast tumor was $2.1 \mathrm{~cm}$, with a maximum of $5.5 \mathrm{~cm}$ and a minimum of $1 \mathrm{~cm}$. The most frequent immunohistochemical subtype was luminal A (39\%), followed by luminal B (36.6\%); the $14.6 \%$ presented a triple negative BC, and 9.7\% had a Her2-enriched BC (Table 1).

The three groups were comparable in terms of age, BMI, and type of breast surgery. However, there was a higher proportion

TABLE 1 | Patients' characteristics.

\begin{tabular}{|c|c|c|c|}
\hline $\begin{array}{l}\text { Breast cancer patients } \\
N=41\end{array}$ & $\begin{array}{l}\text { Group A } \\
N=17\end{array}$ & $\begin{array}{c}\text { Group B } \\
N=7\end{array}$ & $\begin{array}{c}\text { Group C } \\
N=17\end{array}$ \\
\hline Age, years & $55.8 \pm 13.5$ & $55.7 \pm 16.2$ & $59.1 \pm 13.2$ \\
\hline $\mathrm{BMI}, \mathrm{kg} / \mathrm{m}^{2}$ & $24.6 \pm 4.7$ & $25.4 \pm 4.01$ & $25.3 \pm 5.4$ \\
\hline Dyslipidemia & 5 & 2 & 9 \\
\hline Diabetes mellitus & 5 & 1 & 4 \\
\hline \multicolumn{4}{|l|}{ Type of surgery } \\
\hline Mastectomy + AD & 12 & 7 & 14 \\
\hline$B C S+A D$ & 5 & 0 & 3 \\
\hline Tumor size, cm & $2.1 \pm 1.2$ & $2.4 \pm 1.6$ & $1.9 \pm 1.2$ \\
\hline Number of lymph nodes removed & $11.9 \pm 6.9$ & $13 \pm 6.8$ & $8.9 \pm 4.9$ \\
\hline Number of metastatic lymph nodes & $3.2 \pm 1.6$ & $5.1 \pm 7.2$ & $2.1 \pm 1.4$ \\
\hline Neoadjuvant chemotherapy & 4 & 1 & $9^{*}$ \\
\hline \multicolumn{4}{|l|}{ Hystological subtype } \\
\hline Ductal invasive carcinoma & 13 & 6 & 15 \\
\hline Lobular invasive carcinoma & 3 & 1 & 2 \\
\hline Metaplastic carcinoma & 1 & 0 & 0 \\
\hline \multicolumn{4}{|l|}{ Immunoistochemical subtype } \\
\hline Luminal A & 5 & 4 & 7 \\
\hline Luminal B & 9 & 1 & 5 \\
\hline Triple negative & 2 & 1 & 3 \\
\hline Her2 enriched & 1 & 1 & 2 \\
\hline
\end{tabular}

Data are shown as mean $\pm S D$ and $n$.

$A D$, axillary dissection; $B C S$, breast conservative surgery. ${ }^{*} P \leq 0.05$. 
of patients who received neoadjuvant chemotherapy in group $\mathrm{C}$ (52\%) compared to group A (18\%) and group C (52\%) $(p=0.048$; Table 1). Eighty percent of the entire cohort had undergone mastectomy and axillary dissection, while $20 \%$ had undergone breast conservative surgery and axillary dissection.

The median number of lymph nodes removed was 12 (with a maximum of 27 and a minimum of 3 ).

\section{Postoperative Course}

No significant differences were documented among the three groups in terms of total amount of serum drained by the axillary drain within the first 3 postoperative days, and similarly, there was no difference in terms of mean length of hospitalization between patients in the three different groups (Table 2).

When considering the total number of days of the axillary drain maintained at the surgical site, we observed a significant difference among the three groups ( $p=0.02$; Table 2 ). In group $C$, we observed a lower number of days of drain (7 days) compared to group A (14 days) and group B (11 days; Table 2).

The incidence of postoperative seroma was $11.8 \%$ in group $\mathrm{A}$, $14 \%$ in group $\mathrm{B}$, and $6 \%$ in group C ( $p=0.76$; Table 2$)$.

Interestingly, signs of postoperative infection were significantly lower in group $\mathrm{C}$ compared to that in the other two groups $(p=0.02 ; 23 \%$ of patients in group A, $57 \%$ of group B, and $6 \%$ of group C) (Table 2).

Considering the known contribution of a high BMI to an increased potential seroma formation (7), we have additionally investigated a possible correlation between patients' BMI and the total days of permanence of axillary drain, as well as between patients' BMI and quantity of serum drained in the first 3 postoperative days, but we did not find any significant positive correlation either in all the enrolled participants either in each group of participants.

\section{DISCUSSION}

In our study, we observed that, although the use of two different surgical sealants was not associated with a reduction in the incidence of postoperative seroma, the use of cyanoacrylate glue has led to a reduced occurrence of postoperative infections and has allowed a short time of permanence of the axillary drain, maintaining an incidence of seroma development similar to that of the group where no sealant was applied and the drain maintained in the surgical site at least twice the number of days.
The reduction in terms of number of days for the permanence of the axillary drain is certainly beneficial for patients. First, its presence implies the need for repeated outpatient controls. Second, it affects patient's mobility since its location within the surgical wound can give pain and limit movements of the upper limb involved. Moreover, it may also happen that, due to eventual abrupt movements of the limb, the drainage is unknowingly dislocated, causing pain and worry to the patient.

It is also important to consider that any adjuvant therapies (chemotherapy and/or radiotherapy) can be started only when the surgical wound has completely healed; therefore, the permanence of axillary drainage might delay its scheduling (19). In this light, for cancer patients, reducing the rate and duration of complications in the postoperative period represents certainly an advantage.

Similar to the results obtained by Vasileiadou et al. (7), our findings may be in part explained by the fact that the Glubran $₫ 2$ rapidly polymerizes into a thin elastic film, which presents elevated tensile strength and adheres to the tissue once applied. Consequently, the use of this cyanoacrylate glue during surgery may create a sealing coating, which can prevent or reduce seroma formation likely due to a better approximation of wound margins, resulting in a smaller dead volume, and determining a potentially more permanent effect on tissues with respect to the fibrin sealant (12). Cyanoacrylate glue is potentially cytotoxic (20), and it has to be highlighted the importance of training in its application, choosing the right application device, which, for the prevention/treatment of axillary lymphorrhea, consists in nebulizing a small amount of glue through a dedicated nebulizer system in the operating field.

Our study focuses on seroma prevention techniques after axillary dissection, given the relevance of this type of surgery to date. In fact, despite the tendency of BC surgery to an increasingly conservative approach, axillary lymphadenectomy remains an essential intervention in several clinical settings to complete the disease staging (21): in the presence of clinically pathological axillary lymph nodes; in case of preoperative cytological study of lymph nodes with finding of metastatic cells; in the presence of sentinel lymph node histologically positive for macrometastasis; in T4 tumors and inflammatory BC (22); and in case of persistently positive axillary clinical exam following neoadjuvant therapy (21).

Axillary lymphadenectomy may cause several complications, among which the most frequent are seroma, bleeding, and surgical wound infection development $(23,24)$, delaying patient's

TABLE 2 | Postoperative course.

\begin{tabular}{|c|c|c|c|c|}
\hline $\begin{array}{l}\text { Breast cancer patients } \\
N=41\end{array}$ & $\begin{array}{l}\text { Group A } \\
N=17\end{array}$ & $\begin{array}{c}\text { Group B } \\
N=7\end{array}$ & $\begin{array}{l}\text { Group C } \\
N=17\end{array}$ & $P$ \\
\hline Serum quantity drained during the first 3 postoperative days, $\mathrm{ml}$ & $229.1 \pm 124.2$ & $261.4 \pm 132.04$ & $205.8 \pm 123.9$ & 0.77 \\
\hline Axillary drain permanence, days & $14.1 \pm 8.7$ & $11.4 \pm 7.9$ & $7.4 \pm 3.2$ & 0.02 \\
\hline Postoperative seroma incidence, \% & 11.8 & 14 & 6 & 0.76 \\
\hline
\end{tabular}


recovery and any eventual adjuvant therapy. In addition, seroma may lead to limitation in upper limb movements and by postoperative pain $(23,24)$.

In postoperative seroma classification, three degrees are recognized (25): (i) first degree, seroma asymptomatic, evaluable only by ultrasound exam; (ii) second degree, seroma symptomatic that requires outpatient needle aspiration; and (iii) third degree, seroma symptomatic that needs surgical and/or radiological-guided drainage. The pathogenesis of seroma is not yet well-known. Serum accumulates to fill the cavities that are inevitably created following surgical treatment as what happens in lymphadenectomy. Some authors hypothesized that an inflammatory process intervenes in the pathogenesis of seroma, which would result from the damage to vessels and tissues perpetuated during the intervention (19). Seroma may result also as an accumulation of lymph fluids resulting from the lymph nodes dissection (26). Unalp and Onal (27) have investigated the association between seroma development and type of surgery performed, as well as patient's age, weight and comorbidities, and breast volume. The authors found that a drainage flowrate of $>50 \mathrm{ml} /$ day after $48 \mathrm{~h}$ following breast surgery resulted a predicting factor for seroma formation in BC patients, while other investigated variables were not associated with development of seroma (27). Although a significant contribution of a high BMI to increased seroma formation in breast surgery (7) has been reported, a systematic review, conducted to estimate individual risk of seroma formation in breast surgery, found only moderate evidence to support a risk for seroma formation in subjects with higher body weight, extended radical mastectomy as compared with simple mastectomy, and greater drainage volume in the initial postoperative 3 days, but no risk factor to support strong evidence (28).

Regarding the use and management of suction axillary drain in patients undergoing lymphadenectomy, this topic is widely discussed in the literature, but there is no agreement between the numerous experiences. Several studies encourage the use of suction drains and advise against their early removal (before the third postoperative day) (29). A systematic review of randomized controlled trials, which involved 585 BC patients, assessed the incidence of seroma between two groups of patients who underwent axillary dissection, one treated with axillary drain position, and the other one without it (30). The incidence of postoperative seroma was $46.8 \%$ in the first group and $68.8 \%$ in the second group. The review showed that the insertion of a suction drain after axillary dissection reduced seroma development, its incidence, and the frequency of subsequent aspirations (30). On the other hand, this method increases the hospitalization length, even if experiences of early discharge with onsite drain and its outpatient management have been described. These data have been confirmed by a Cochrane review of randomized controlled trials involving 960 patients undergoing axillary dissection, with or without drain insertion (31). Ten Wolde et al. in 2014 (32) proposed, as an alternative to the use of drains following mastectomy, the use of the quilting suture, consisting in the mechanical occlusion of space through sutures along the surgical site that secure the subcutaneous tissue to the pectoral and/or dentate muscle fascia. This technique, by reducing dead spaces, resulted in the effective prevention of seroma (32). Garbay et al. described the use of quilting sutures also following axillary dissection (33). The authors observed a reduction in terms of average length of hospitalization, need for seroma aspiration, and intensity of postoperative pain (33). It has also been described by Mazouni et al. (34) the use of quilting sutures in association with suction drain for the prevention of postmastectomy seroma, which resulted in a reduction in the rate of postoperative seroma development, volume of drained seroma, and length of drain maintenance in the surgical site.

The use of fibrin sealant as hemostatic agents in surgery is well-documented, although there are controversial results on its ability to reduce fluid accumulation in dissected spaces through tissue adhesion, as in inguinal hernia repair (35). Fibrin sealants are biological hemostatic agents that interact with tissues during surgery, promoting hemostasis, growth, and duplication of fibroblasts, and favoring closure of lymphatic vessels. Furthermore, fibrin sealant likely may mechanically obliterate the dead space where serous fluid accumulates after surgical dissection (4). Nevertheless, some studies have evaluated the use of fibrin sealant in addition (36) or in replacement (4) to the drain position, resulting in a lower hospital stay length and lower costs but not modifying significantly the incidence of postoperative seroma in the group of patients treated with fibrin sealant. Similarly, Burak et al. (37) and Langer et al. (38) documented no difference in the incidence of seroma after axillary lymph nodes dissection when using this agent, in accordance with our results. It has been hypothesized that cyanoacrylate adhesive glue may have longer and more permanent effect on tissues than the frequently used fibrin sealant, due to its properties (7). The use of cyanoacrylate glue has been described in general and laparoscopic surgery. Good results have been obtained in the treatment of acute bleeding of gastric (16) and esophageal varices (17), for the treatment of external pancreatic fistulas (39), and for mesh fixation during laparoscopic inguinal hernioplasty (18). The use of cyanoacrylate has also been evaluated in the prevention of postoperative lymphocele after lymphadenectomy in patients with uterine tumor (15), showing that in the cyanoacrylate group, only $15 \%$ of the patients developed lymphocele vs. $36 \%$ in the control group. The volume of lymphorrhea was lower in the cyanoacrylate group with respect to the control group (15). Vasileiadou et al. (7) obtained interesting results using the cyanoacrylate glue in association with the suction drainage: it seemed to contribute to the reduction in the onset of seroma, with a lower need for evacuative punctures and a reduction in terms of number of permanence days of the axillary drain. The assumed mechanism of action is that its adhesive property may create a sealing coating in the surgical field, occluding lymphatic leaks and limiting the accumulation of seroma (7), determining potential positive effects.

Considering the results of our study, some limitations have to be acknowledged. We conducted a retrospective study, with access only to clinical data that are normally collected within routine clinical practice and with lack of methodological rigor; in fact, the sample size in the different groups was not homogeneous, with fewer patients in group B compared to 
groups A and C. Moreover, in group B, the presence of axillary suction drain might have compromised the stabilization of fibrin clots provided by the fibrin sealant and have interfered with adherence between tissues. Also in this setting, choosing the right spray during training for its application is important. The greater obstacle when using fibrin sealant is the mode of application in order to allow adequate activation of the glue and reach the solid phase.

In our retrospective evaluation, we found no evidence that the use of surgical glues might actually reduce the formation of seroma following axillary dissection, and in this light, each group size did not allow a reliable conclusion on this aspect.

However, we have been able to observe that the use of cyanoacrylate glue in association with the positioning of an axillary closed suction drain in patients undergoing axillary dissection may contribute to the reduction in the length of drain permanence and the incidence of infections in the postoperative period.

It would be interesting to deepen our experience by conducting further prospective and multicenter studies in this regard.

\section{REFERENCES}

1. Veronesi U, Stafyla V, Luini A, Veronesi P. Breast cancer: from "maximum tolerable" to "minimum effective" treatment. Front Oncol. (2012) 2:125. doi: 10.3389/fonc.2012.00125

2. Tardioli S, Ballesio L, Gigli S, DI Pastena F, D’Orazi V, Giraldi G, et al. Wireguided Localization in non-palpable breast cancer: results from monocentric experience. Anticancer Res. (2016) 36:2423-7.

3. Amabile MI, Mazouni C, Guimond C, Sarfati B, Leymarie N, Cloutier AS, et al. Factors predictive of re-excision after oncoplastic breast-conserving surgery. Anticancer Res. (2015) 35:4229-34.

4. Conversano A, Mazouni C, Thomin A, Gaudin A, Fournier M, Rimareix F, et al. Use of low-thrombin fibrin sealant glue after axillary lymphadenectomy for breast cancer to reduce hospital length and seroma. Clin Breast Cancer. (2017) 17:293-7. doi: 10.1016/j.clbc.2016.12.013

5. Srivastava V, Basu S, Shukla VK. Seroma formation after breast cancer surgery: what we have learned in the last two decades. J Breast Cancer. (2012) 15:37380. doi: 10.4048/jbc.2012.15.4.373

6. Sampathraju S, Rodrigues G. Seroma formation after mastectomy: pathogenesis and prevention. Indian J Surg Oncol. (2010) 1:328-33. doi: 10.1007/s13193-011-0067-5

7. Vasileiadou K, Kosmidis C, Anthimidis G, Miliaras S, Kostopoulos I, Fahantidis, et al. Cyanoacrylate adhesive reduces seroma production after modified radical mastectomy or quadrantectomy with lymph node dissectiona prospective randomized clinical trial. Clin Breast Cancer. (2017) 17:595600. doi: 10.1016/j.clbc.2017.04.004

8. Divino CM, Kuerer HM, Tartter PI. Drains prevent seromas following lumpectomy with axillary dissection. Breast J. (2000) 6:31-3. doi: 10.1046/j.1524-4741.2000.99037.x

9. Rice DC, Morris SM, Sarr MG, Farnell MB, van Heerden JA, Grant CS, et al. Intraoperative topical tetracycline sclerotherapy following mastectomy: a prospective, randomized trial. J Surg Oncol. (2000) 73:224-7. doi: 10.1002/ (SICI)1096-9098(200004)73:4<224::AID-JSO7>3.0.CO;2-0

10. Chaturvedi P, Chaturvedi U. Axillary compression with delayed drain removal reduces prolonged seroma formation. J Surg Oncol. (2001) 78:27980. doi: 10.1002/jso.1168

11. Huang J, Yinghua Y, Changyuan W, Qin Q, Mo Q, Yang W. Harmonic scalpel versus electrocautery dissection in modified radical mastectomy for breast cancer: a meta analysis. PLoS One. (2005) 10:e0142271. doi: 10.1371/journal.pone.0142271

\section{DATA AVAILABILITY STATEMENT}

The raw data supporting the conclusions of this article will be made available by the authors, without undue reservation.

\section{ETHICS STATEMENT}

The studies involving human participants were reviewed and approved by Comitato Etico dell'Università "Sapienza," AOU Policlinico Umberto I, Rome, Italy. The patients/participants provided their written informed consent to participate in this study.

\section{AUTHOR CONTRIBUTIONS}

$\mathrm{AD}$ and DT conducted research, analyzed data, and wrote the paper. FF conducted research. BL conducted research and analyzed data. $\mathrm{BC}, \mathrm{AB}, \mathrm{MV}, \mathrm{VD}$ 'A, and $\mathrm{DP}$ reviewed the paper. SS wrote and reviewed the paper. MA designed and conducted the research and wrote and reviewed the paper. All authors read and approved the final manuscript.

12. Dinsmore RC, Harris JA, Gustafson RJ. Effect of fibrin glue on lymphatic drainage after modified radical mastectomy: a prospective randomized trial. Am Surg. (2000) 66:982-985.

13. Docimo G, Limongelli P, Conzo G, Gili S, Bosco A, Rizzuto A, et al. Axillary lymphadenectomy for breast cancer elderly patients and fibrin glue. BMC Surg. (2013) 13:S8. doi: 10.1186/1471-2482-13-S2-S8

14. Gisquet H, Delay E, Paradol P, Toussoun G, Delaporte T, Perol D. Prevention of seroma by quilting suture after harvesting latissimus dorsi flap. The "Chippendale" technique. Ann Chir Plast Esthet. (2010) 55:97103. doi: 10.1016/j.anplas.2009.05.002

15. Bifulco G, Giampaolino P, Morra I, Tommaselli GA, Tarsitano F, Chiacchio G, et al. Synthetic cyanoacrylic glue in the prevention of post-operative lymphocele after pelvic lymphadenectomy in patients with uterine malignancies: a prospective, single-blind, preliminary study. Gynecol Oncol. (2014) 134:556-60. doi: 10.1016/j.ygyno.2014. 06.015

16. Seewald S, Sriram PVJ, Naga M, Fennerty MB, Boyer J, Oberti F, et al. Cyanoacrylate glue in gastric variceal bleeding. Endoscopy. (2002) 34:92632. doi: 10.1055/s-2002-35312

17. Ayyildiz SN, Ayyildiz A. Cyanoacrylic tissue glues: Biochemical properties and their usage in urology. Turk J Urol. (2017) 43:14-24. doi: 10.5152/tud.2017.09465

18. Lasheen AE, Tolba AM, Mohamed H, Mohammed H, Smaeil NA. Laparoscopic inguinal hernia repair with closure of hernial defect and central mesh fixation using Glubran 2. Surg Sci. (2013) 4:554-7. doi: 10.4236/ss.2013.412107

19. McCaul JA, Aslaam A, Spooner RJ, Louden I, Cavanagh T, Purushotham AD. Aetiology of seroma formation in patients undergoing surgery for breast cancer. Breast. (2000) 9:144-8. doi: 10.1054/brst.199 9.0126

20. Pascual G, Sotomayor S, Rodríguez M, Pérez-Köhler B, Kühnhardt A, Fernández-Gutiérrez $\mathrm{M}$, et al. Cytotoxicity of cyanoacrylate-based tissue adhesives and short-term preclinical in vivo biocompatibility in abdominal hernia repair. PLoS One. (2016) 11:e0157920. doi: 10.1371/journal.pone.01 57920

21. Performance and Practice Guidelines for Axillary Lymph Node Dissection in Breast Cancer Patients. The American Society of Breast Surgeons, Guidelines Approved November 25, 2014 (2014).

22. Pasta V, Mitri F, Amabile MI, Picardi N. Inflammatory breast cancer. Ann Ital Chir. (2006) 77:275-9. 
23. Lin PP, Allison DC, Wainstock J, Miller KD, Dooley WC, Friedman N, et al. Impact of axillary lymph node dissection on the therapy of breast cancer patients. J Clin Oncol. (1993) 11:1536-44. doi: 10.1200/JCO.1993.11.8.1536

24. Reynolds JV, Mercer P, McDermott EWM, Cross S, Stokes M, Murphy D, et al. Audit of complete axillary dissection in early breast cancer. Eur J Cancer. (1994) 30:148-9. doi: 10.1016/0959-8049(94)90075-2

25. Kuroi K, Shimozuma K, Taguchi T, Imai H, Yamashiro H, Ohsumi S, et al. Pathophysiology of seroma in breast cancer. Breast Cancer. (2005) 12:28893. doi: $10.2325 /$ jbcs. 12.288

26. Petrek JA, Peters MM, Nori S, Knauer C, Kinne DW, Rogatko A. Axyllary lymphadenectomy. A prospective, randomized trial of 13 factors influencing drainage, including early or delayed arm mobilization. Arch Surg. (1990) 125:378-82. doi: 10.1001/archsurg.1990.01410150100018

27. Unalp HR, Onal MA. Analysis of risk factors affecting the development of seromas following breast cancer surgerier: seromas following breast cancer surgeries. Breast J. (2007) 13:588-92. doi: 10.1111/j.1524-4741.2007.00509.x

28. Kuroi K, Shimozuma K, Taguchi T, Imai H, Yamashiro H, Ohsumi S, et al. Evidence-based risk factors for seroma formation in breast surgery. Jpn J Clin Oncol. (2006) 36:197-206. doi: 10.1093/jjco/hyl019

29. Barton A, Blitz M, Callahan D, Yakimets W, Adams D, Dabbs K. Early removal of postmastectomy drains is not beneficial: results from a halted randomized controlled trial. Am J Surg. (2006) 191:652. doi: 10.1016/j.amjsurg.2006.01.037

30. He XD, Guo ZH, Tian JH, Yang KH, Xie XD. Whether drainage should be used after surgery for breast cancer? A systematic review of randomized controlled trials. Med Oncol. (2011) 28:S22-S30. doi: 10.1007/s12032-0109673-2

31. Thomson DR, Sadideen H, Furniss D. Wound drainage after axillary dissection for carcinoma of the breast. Cochrane Database Syst Rev. (2013) 10:CD006823. doi: 10.1002/14651858.CD006823.pub2

32. ten Wolde B, van den Wildenberg FJH, Keemers-Gels ME, Polat F, Strobbe LJ. Quilting prevents seroma formation following breast cancer surgery: closing the dead space by quilting prevents seroma following axillary lymph node dissection and mastectomy. Ann Surg Oncol. (2014) 21:8027. doi: 10.1245/s10434-013-3359-x

33. Garbay JR, Thoury A, Moinon E, Cavalcanti A, Palma MD, Karsenti G, et al. Axillary padding without drainage after axillary lymphadenectomy - a prospective study of 299 patients with early breast cancer. Breast Care. (2012) 7:231-5. doi: 10.1159/000341102

34. Mazouni C, Mesnard C, Cloutier AS, Amabile MI, Bentivegna E, Garbay JR, et al. Quilting sutures reduces seroma in mastectomy. Clin Breast Cancer. (2015) 15:289-93. doi: 10.1016/j.clbc.2014.12.014

35. Massey LH, Pathak S, Bhargava A, Smart NJ, Daniels IR. The use of adjuncts to reduce seroma in open incisional hernia repair: a systematic review. Hernia. (2018) 22:273-83. doi: 10.1007/s10029-017-1690-z

36. Kain PK, Sowdi R, Anderson ADG, MacFie J. Randomized clinical trial investigating the use of drains and fibrin sealeant following surgery for breast cancer. Br J Surg. (2004) 91:54-60. doi: 10.1002/bjs.4435

37. Burak WE, Goodman PS, Young DC, Farrar WB. Seroma formation following axillary dissection for breast cancer: risk factors and lack of influence of bovine thrombin. J Surg Oncol. (1997) 64:27-31. doi: 10.1002/(SICI)10969098(199701)64:1<27::AID-JSO6>3.0.CO;2-R

38. Langer S, Guenther M, Fronzo A. Does fibrin sealeant reduce drain output and allow earlier removal of drainage cathter in women undergoing operation for breast cancer? Am Surg. (2003) 69:77-81.

39. Mutignani M, Tringali A, Khodadadian E, Petruzziello L, Spada C, Spera G, et al. External pancreatic fistulas resistant to conventional endoscopic therapy: endoscopic closure with N-butyl-2-Cyanoacrylate (Glubran 2). Endoscopy. (2004) 36:738-42. doi: 10.1055/s-2004825672

Conflict of Interest: The authors declare that the research was conducted in the absence of any commercial or financial relationships that could be construed as a potential conflict of interest.

The handling editor declared a shared affiliation with the authors.

Copyright (C) 2020 De Luca, Tripodi, Frusone, Leonardi, Cerbelli, Botticelli, Vergine, D'Andrea, Pironi, Sorrenti and Amabile. This is an open-access article distributed under the terms of the Creative Commons Attribution License (CC BY). The use, distribution or reproduction in other forums is permitted, provided the original author(s) and the copyright owner(s) are credited and that the original publication in this journal is cited, in accordance with accepted academic practice. No use, distribution or reproduction is permitted which does not comply with these terms. 